\section{N-Acetyl Aspartate}

Sarah A. Raskin

Department of Psychology and Neuroscience

Program, Trinity College, Hartford, CT, USA

\section{Synonyms}

Acetylaspartic acid; NAA

\section{Definition}

$\mathrm{N}$-acetylaspartic acid (NAA) is synthesized in the brain from the amino acid aspartic acid and acetyl coenzyme A. NAA is present exclusively in the nervous system and is one of the most highly concentrated chemicals in the brain. It is concentrated in neuron cell bodies but also in oligodendrocytes. NAA is involved in fluid balance in the brain, serves as a source of acetate for myelin synthesis in oligodendrocytes, is a precursor for the synthesis of $\mathrm{N}$-acetylaspartylglutamate, and contributes to energy production in neuronal mitochondria.

\section{Current Knowledge}

NAA is used in studies using magnetic resonance spectroscopy imaging because it emits the strongest signal in the brain, and NAA levels are decreased in many neuropathological conditions, including traumatic brain injury, epilepsy, schizophrenia, and Alzheimer's disease. It is unknown if changes in NAA levels in these conditions are etiological or secondary to other factors. Canavan's disorder is a congenital disorder of NAA metabolism. Increased levels of NAA have been reported to be related to working memory performance (Kozlovskiy et al. 2012).

\section{Cross-References}

$\checkmark$ Functional Imaging

Functional Magnetic Resonance Imaging

\section{References and Readings}

Benarroch, E. (2008). $N$-acetylaspartate and $\mathrm{N}$-acetylaspartylglutamate: Neurobiology and clinical significance. Neurology, 70, 1353-1357.

Kozlovskiy, S., Vartanov, A., Pyasik, M., \& Polikanova, I. (2012). Working memory and $N$-acetylaspartate level in hippocampus, parietal cortex and subventricular zone. International Journal of Psychology, 47, 584.

Moffat, J., Ross, B., Peethambaran, A., Madhavarao, C., \& Namboodiri, A. (2007). $N$-acetylaspartate in the CNS: From neurodiagnostics to neurobiology. Progress in Neurobiology, 81, 89-131.

Moffett, J., Tieman, S., Weinberger, D., Coyle, J., \& Namboodiri, A. (2006). $N$-acetylaspartate: A unique neuronal molecule in the central nervous system. New York: Springer. 